\title{
Percussion Synthesis Based on Models of Nonlinear Shell Vibration
}

\author{
Stefan Bilbao
}

\begin{abstract}
The synthesis of sound based on physical models of 2-D percussion instruments is problematic and has been approached only infrequently in the literature. Beyond the computational expense inherent to the simulation of 2-D systems, a deeper difficulty is in dealing with the strong nonlinearity exhibited by thin structures when struck-this nonlinearity leads to phenomena which are not captured, even approximately, by a linear model, and nearly all synthesis work is based on the assumption that the distributed resonating component of a musical instrument is linear. Perceptually, the effects of the vibration of a thin structure at high amplitudes can be heard as crashes, pitch glides, and the slow buildup of high-frequency energy characteristic of gongs. A large family of instruments may be described, approximately, as circular thin shells, of approximately spherical geometry, in which case a tractable PDE description, described here, is available. Time-domain finite-difference schemes, in radial coordinates, are a suitable method for synthesis. Stability conditions, numerical boundary conditions both at the edge and center, and implementation details are discussed, and simulation results are presented, highlighting the various perceptual effects mentioned above.
\end{abstract}

Index Terms-Cymbals, finite-difference schemes, gongs, musical acoustics, nonlinear distributed systems, percussion, physical modeling, sound synthesis.

\section{INTRODUCTION}

$\mathbf{P}$ HYSICAL modeling synthesis techniques have been applied to a wide variety of instruments, including those of the string, brass, and woodwind families, as well as the human voice. Many techniques have evolved-perhaps the best known are digital waveguides [1] and modal synthesis [2]. Synthesis from models of percussion instruments, however, has seen somewhat less investigation, which is not surprising, given that the body of musical acoustics research into such instruments is relatively small. See [3] and [4] for an overview. Nevertheless, various synthesis techniques have developed. The digital waveguide mesh is often used in the case of the linear vibration of membranes [5]-[8], and plates [9], and modal synthesis techniques have been applied to both such systems; related to modal synthesis is a method based on transfer function methods, and suited to linear problems, known as the

Manuscript received March 31, 2009; revised July 20, 2009. Current version published April 14, 2010. This work was supported by the Engineering and Physical Sciences Research Council, U.K., under Grant C007328/1. The associate editor coordinating the review of this manuscript and approving it for publication was Dr. Federico Fontana.

The author is with the Acoustics and Fluid Dynamics Group/Music subject area, University of Edinburgh, Edinburgh EH9 3JZ, U.K. (e-mail: sbilbao@staffmail.ed.ac.uk).

Digital Object Identifier 10.1109/TASL.2009.2029710 functional transformation method [10] which has also been applied to linear 2-D structures, including those exhibiting a tension-modulation nonlinearity [11]. For 2-D systems, computational complexity is relatively large-until recently, real- or near real-time synthesis on desktop hardware has not been possible, which is perhaps another way of explaining the relative paucity of work on synthetic percussion.

The issue of computational complexity is, at this point, near to being resolved, due to the great advances in computing power in recent years, making a reappraisal of such synthesis techniques timely. A serious difficulty, though, presents itself in the distributed nonlinear nature of some such instruments-such behavior is of crucial perceptual importance, leading to effects such as pitch glides, crashes, and the slow buildup of high-frequency energy as heard in, e.g., gongs, and is not adequately captured by linear approximations, such as those which lead to typical waveguide and modal formulations. (Nonlinear models of tension modulation type can capture the first of these effects but not the others.) A direct time/space domain formulation, based on a suitable nonlinear plate or shell model is a good starting point. In the case of irregular geometries, finite-element methods are ideal, but given that various percussion instruments, such as cymbals and gongs, are well-described in a simple radial coordinate system, finite-difference methods are a relatively simple alternative. Finite-difference schemes for the linear vibration and reverberation of rectangular plates have been explored by various authors [12]-[14], and the nonlinear case has been described in [15].

The present paper is concerned with the extension of such techniques to the case of curved shells, and thus to modeling of instruments such as cymbals and gongs, though there is an emphasis on general techniques here rather than of modeling of a specific instrument. A model of nonlinear shell vibration is presented in Section II, followed by a suitable finite-difference scheme in Section III, accompanied by a discussion of stability conditions, numerical boundary and center conditions, input and output, and implementation details. Simulation results are presented in Section IV. Sound examples are available on the author's website at http://ccrma.stanford.edu/ bilbao/soundex/ shell/

Some of the material here will soon appear in a very abbreviated form in [16]; the present paper contains a much expanded treatment of implementation details, computational complexity, numerical dispersion, and bandlimiting, and simulation results.

\section{SPHERICAL SHELl ViBRATION}

Percussion instruments, such as cymbals and gongs are thin, curved metallic structures. As such, they may be described as shells. In the case of a general such structure, there is a very 


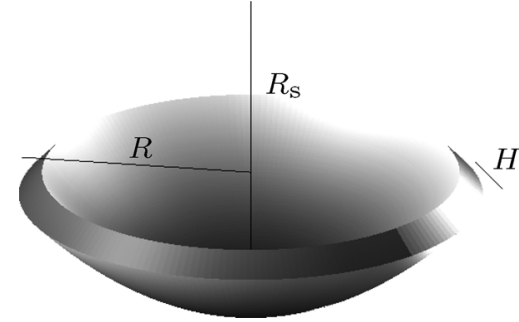

Fig. 1. Spherical cap, of radius of curvature $R_{\mathrm{S}}$, thickness $H$, and outer radius $R$.

large variety of models available—see, e.g., [17]. If the structure is very thin, and the curvature is not extreme, and, furthermore, the shell is approximately a spherical cap, then tractable models suitable for sound synthesis applications are available. One such model, used in studies of such instruments [18] is similar to the following:

$$
\begin{aligned}
\rho H u_{t t}= & -D \Delta \Delta u+\mathcal{L}(\Phi, u)-\frac{1}{R_{\mathrm{S}}} \Delta \Phi \\
& -2 \sigma_{0} \rho H u_{t}+2 \sigma_{1} \rho H \Delta u_{t}+f \epsilon \\
\Delta \Delta \Phi= & -\frac{E H}{2} \mathcal{L}(u, u)+\frac{E H}{R_{\mathrm{S}}} \Delta u
\end{aligned}
$$

Here, $u$ is the transverse displacement of the shell, as a function of radius $r$, angle $\theta$, and time $t$, and $\Phi$ is an auxiliary function, sometimes referred to as the Airy stress function. $\rho$ is material density, $H$ is the thickness of the shell, $E$ is Young's modulus, and $\sigma_{0}$ and $\sigma_{1}$ are loss parameters (the second of which gives rise to frequency-dependent loss, and which is not modeled in [18]). The parameter $D$ is defined as $D=E H^{3} / 12\left(1-\nu^{2}\right)$, and is also dependent on $\nu$, Poisson's ratio for the material. $R_{s}$ is the radius of curvature of the shell. The system is assumed defined over $r \in[0, R], \theta \in[0,2 \pi), t \geq 0$. See Fig. 1 . The subscript $t$ refers to partial time differentiation.

The operator $\Delta$ is the Laplacian, defined, in radial coordinates, for a given function $f(r, \theta, t)$, as

$$
\Delta f=\frac{1}{r}\left(r f_{r}\right)_{r}+\frac{1}{r^{2}} f_{\theta \theta}
$$

where subscripts $r$ and $\theta$ refer to partial differentiation with respect to $r$ and $\theta$, respectively. The operator $\Delta \Delta$, a double application of the Laplacian operator, is often referred to as a biharmonic operator or bi-Laplacian.

The nonlinear operator $\mathcal{L}(\alpha, \beta)$ is defined, in terms of two functions $\alpha(r, \theta, t)$ and $\beta(r, \theta, t)$, as

$$
\begin{aligned}
\mathcal{L}(\alpha, \beta)=\frac{\alpha_{r r}}{r}\left(\beta_{r}+\frac{\beta_{\theta \theta}}{r}\right)+\frac{\beta_{r r}}{r}\left(\alpha_{r}+\frac{\alpha_{\theta \theta}}{r}\right) & \\
& -\frac{2}{r^{2}}\left(\alpha_{r \theta}-\frac{\alpha_{\theta}}{r}\right)\left(\beta_{r \theta}-\frac{\beta_{\theta}}{r}\right) .
\end{aligned}
$$

The term $f \epsilon$, where $f=f(t)$ and $\epsilon=\epsilon(r, \theta)$ represents an excitation due to a force $f=f(t)$ assumed to act on the shell according to a fixed spatial distribution $\epsilon=\epsilon(r, \theta)$. $f$ could result from coupling to a model of a striking element such as a mallet, or, in the simplest case, may be specified as a given control signal. $\epsilon$ is normally sharply peaked.
In the limit of low curvature (i.e., as $R_{\mathrm{S}}$ becomes large), and under lossless conditions, system (1) reduces to the von Kármán system [19], [20], describing nonlinear vibration of a thin flat plate; at low vibration amplitudes (i.e., when the terms involving the operator $\mathcal{L}$ become negligible), the system decouples, resulting in the usual linear equation of vibration of a thin plate [21].

\section{A. Scaled Form}

As a prelude to developing a synthesis algorithm, it is useful to non-dimensionalize the dependent and spatial variables of the system, through the introduction of the variables $r^{\prime}=r / R$, $u^{\prime}=u / u_{0}, \Phi^{\prime}=\Phi / \Phi_{0}$, where

$$
u_{0}=H / \sqrt{6\left(1-\nu^{2}\right)} \quad \Phi_{0}=D
$$

leading, after removal of primes, to the system

$$
\begin{aligned}
u_{t t}= & -\kappa^{2}(\Delta \Delta u-\mathcal{L}(\Phi, u)+q \Delta \Phi) \\
& -2 \sigma_{0} u_{t}+2 \sigma_{1} \Delta u_{t}+F \epsilon \\
\Delta \Delta \Phi= & -\mathcal{L}(u, u)+q \Delta u
\end{aligned}
$$

where the two parameters $\kappa$ and $q$ are defined as

$$
\kappa=\sqrt{\frac{D}{\rho H R^{4}}} \quad q=\frac{R^{2} \sqrt{6\left(1-\nu^{2}\right)}}{H R_{s}} .
$$

The shell is now defined over the unit disc, $0 \leq r \leq 1$. $F(t)$ is defined as $F=f / \rho H u_{0}$.

The parameter $\kappa$ scales roughly with pitch (though the spectrum of sounds produced from a shell model is necessarily strongly inharmonic), and, for musical systems, often lies in a range between 10 and 150. $q$ summarizes the effects of curvature, and normally takes on values between 0 (i.e., when the shell is flat), and approximately 100 . The spectral effect of curvature will be examined in Section IV-B.

\section{B. Decay Time}

Decay time is frequency-dependent in this model, and can be shown to be (under linear conditions, and in the limit of high frequencies) approximately

$$
T_{60}(f)=\frac{6 \ln (10)}{\sigma_{0}+2 \pi f \sigma_{1} / \kappa}
$$

where $T_{60}(f)$ is the $60-\mathrm{dB}$ decay time of a component of frequency $f$. The model of loss as presented here is an ad hoc one, which captures the essential feature of increased damping at high frequencies, and has the virtue of simplicity, but more elaborate models are available [22]. Frequency-dependent loss is more important in the nonlinear case than in the linear case, as effects such as pitch glides and crashes are strongly influenced by damping rates—-see Sections IV-C and IV-D.

\section{Edge and Center Conditions}

In many percussion configurations, the edge of the shell at $r=1$ is free to vibrate; free boundary conditions in radial coor- 


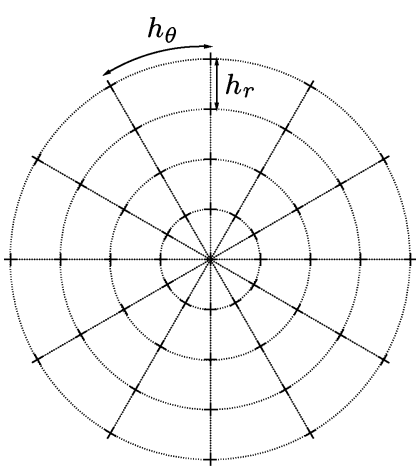

(a)

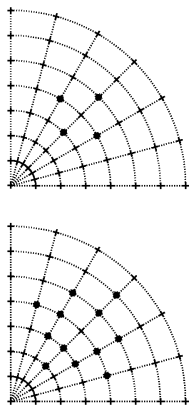

(b)
Fig. 2. (a) Radial grid, of radial grid spacing $h_{r}$, and angular spacing $h_{\theta}$, and (b) stencils of the operators $\delta_{\Delta}$ (top) and $\delta_{\Delta \Delta}$ (bottom), where at a given operating point, indicated by a cross, values at the points indicated by black circles are required.

dinates [18] are similar to those used in the case of flat circular plates [23]. For the variable $u$, the conditions are

$$
\begin{aligned}
u_{r r}+\nu u_{r}+\nu u_{\theta \theta} & =0 \\
u_{r r r}+u_{r r}-u_{r}+(\nu-3) u_{\theta \theta}+(2-\nu) u_{r \theta \theta} & =0
\end{aligned}
$$

and there is thus an explicit dependence on Poisson's ratio, which must be included in a simulation as an extra global parameter in addition to $\kappa$ and $q$ as defined above. (Note also that $\kappa$ is defined in terms of $\nu$.) In the remainder of this paper, it will be assumed to be equal to 0.3 , the value for steel, and values for other materials are not extremely different. For the Airy stress function, a simple boundary condition is

$$
\Phi=\Phi_{r}=0
$$

which may be shown to be equivalent to other forms of the free boundary condition which appear in the literature [18] (notice that only higher derivatives of $\Phi$ appear in system (5), and there is thus some flexibility in setting boundary conditions [16]).

In some instruments, such as gongs, the boundary condition is perhaps better modeled as clamped, or simply supported [4], due to the presence of a heavy rim - this poses no new difficulties, numerically, as termination using such conditions is simpler than using the above free conditions.

If the shell center is unconstrained, then no further conditions are necessary. If, however, the shell is attached to a supporting structure at its center, then clearly a fixed condition of some form is required near $r=0$. A simple (and perhaps not extremely realistic) assumption is that the shell is clamped over a circle of radius $\eta \ll 1$, i.e.,

$$
u=u_{r}=\Phi=\Phi_{r}=0 \quad \text { at } \quad r=\eta
$$

This is a good approximation in the case of, e.g., a high-hat cymbal. Other center conditions are possible-for instance, one might employ a pivoting condition in the case of, e.g., a ride cymbal. See Section V for more comments on this subject.

\section{TIME-DomaIn METHODS}

\section{A. Grid Functions and Difference Operators in Radial Coordinates}

For a continuous problem defined over a circle of radius 1 , such as the scaled system (5), a grid function $f_{l, m}^{n}$ approximates a continuous function $f(r, \theta, t)$, at times $t=n h_{t}$, for $n \geq 0$, and at locations $r=l h_{r}$ and $\theta=m h_{\theta}$, for $l=0 \ldots, N_{r}$, and $m=0 \ldots, N_{\theta}-1$. Here $h_{t}$ is a time step (and $f_{\mathrm{S}}=1 / h_{t}$ is the sample rate, typically chosen $a$ priori as an audio rate such as $32,44.1$, or $48 \mathrm{kHz}$ ) and $h_{r}$ and $h_{\theta}$ are grid spacings in the radial and angular directions respectively. The grid spacings are chosen here such that $h_{r}=1 / N_{r}$ and $h_{\theta}=2 \pi / N_{\theta}$ for some integers $N_{r}$ and $N_{\theta}$. At the central grid point, at $l=0$, the grid function is assumed uniquely defined, and the value at this location is written as $f_{0,0}^{n}$. See Fig. 2(a).

Forward and backward unit shifts in the time direction, and in the coordinate directions $r$ and $\theta$ may be defined as

$$
\begin{array}{ll}
e_{t+} f_{l, m}^{n}=f_{l, m}^{n+1} & e_{t-} f_{l, m}^{n}=f_{l, m}^{n-1} \\
e_{r+} f_{l, m}^{n}=f_{l+1, m}^{n} & e_{r-} f_{l, m}^{n}=f_{l-1, m}^{n} \\
e_{\theta+} f_{l, m}^{n}=f_{l, m+1}^{n} & e_{\theta-} f_{l, m}^{n}=f_{l, m-1}^{n}
\end{array}
$$

There are some restrictions on the use of these operators due to the choice of coordinate system, namely that the backward radial shift operator $e_{r}-$ may only be applied at grid locations $l>0$ (i.e., not at the center grid point), and that the shifts in the angular direction must be taken modulo $N_{\theta}$, i.e., $e_{\theta+} f_{l, N_{\theta}-1}^{n}=$ $f_{l, 0}^{n}$.

Basic difference operators may be defined in terms of these shifts. For any coordinate $\alpha$

$$
\begin{aligned}
\delta_{\alpha+} & =\frac{1}{h_{\alpha}}\left(e_{\alpha+}-1\right) \quad \delta_{\alpha-}=\frac{1}{h_{\alpha}}\left(1-e_{\alpha-}\right) \\
\delta_{\alpha \alpha} & =\frac{1}{h_{\alpha}^{2}}\left(e_{\alpha+}-2+e_{\alpha-}\right) \quad \delta_{\alpha \cdot}=\frac{1}{2 h_{\alpha}}\left(e_{\alpha+}-e_{\alpha-}\right) \\
\mu_{\alpha+} & =\frac{1}{2}\left(e_{\alpha+}+1\right) \quad \mu_{\alpha-}=\frac{1}{2}\left(1+e_{\alpha-}\right)
\end{aligned}
$$

where $\delta_{\alpha+}, \delta_{\alpha-}$ and $\delta_{\alpha}$. are forward, backward, and centered approximations to a first derivative, and where $\delta_{\alpha \alpha}$ is an approximation to a second derivative. $\mu_{\alpha+}$ and $\mu_{\alpha-}$ are forward and backward averaging operators, respectively.

An approximation $\delta_{\Delta}$ to the Laplacian $\Delta$, as defined in (2) may be defined, at grid points with $l>0$, as

$$
\delta_{\Delta}=\delta_{r r}+\frac{1}{r} \delta_{r}+\frac{1}{r^{2}} \delta_{\theta \theta} .
$$

An approximation to the Laplacian at the central grid point with $l=0$ (necessary in this context if the shell is unconstrained at the center) is [24]:

$$
\delta_{\Delta} f_{0,0}=\frac{4}{N_{\theta} h_{r}^{2}} \sum_{m=0}^{N_{\theta}-1} f_{1, m}^{n}-f_{0,0}^{n} .
$$

The biharmonic operator $\Delta \Delta$ may be approximated, at grid points with $l>1$, through a double application of this $\delta_{\Delta}$, i.e., through the use of the operator $\delta_{\Delta \Delta}=\delta_{\Delta} \delta_{\Delta}$. At grid points 
with $l=1$, and at the central grid point $l=0$, special forms are again necessary:

$$
\begin{aligned}
\delta_{\Delta \Delta} f_{0,0}^{n} & =\frac{16}{3 N_{\theta} h_{r}^{4}} \sum_{m=0}^{N_{\theta}-1}\left(f_{2, m}^{n}-4 f_{1, m}^{n}+3 f_{0,0}^{n}\right) \\
\delta_{\Delta \Delta} f_{1, m}^{n} & =\frac{4}{N_{\theta} h_{r}^{2}} \sum_{m=0}^{N_{\theta}-1} \delta_{\Delta} f_{1, m}^{n}-\delta_{\Delta} f_{0,0}^{n} .
\end{aligned}
$$

Slightly different formulations have appeared in the literature [12].

The other important differential operator in (5) is the bilinear operator $\mathcal{L}$. An approximation $\mathfrak{l}(\alpha, \beta)$, applied to two grid functions $\alpha_{l, m}^{n}$ and $\beta_{l, m}^{n}$, at grid points with $l>0$ is

$$
\begin{aligned}
\mathfrak{l}(\alpha, \beta)= & \frac{\delta_{r r} \alpha}{r}\left(\delta_{r} \cdot \beta+\frac{\delta_{\theta \theta} \beta}{r}\right)+\frac{\delta_{r r} \beta}{r}\left(\delta_{r} \cdot \alpha+\frac{\delta_{\theta \theta} \alpha}{r}\right) \\
& -\frac{2}{r^{2}}\left(\delta_{r} \cdot \delta_{\theta} \cdot \alpha-\frac{\delta_{\theta} \cdot \alpha}{r}\right)\left(\delta_{r} . \delta_{\theta} \cdot \beta-\frac{\delta_{\theta \cdot} \beta}{r}\right) .
\end{aligned}
$$

A special form is again necessary if an approximation to $\mathcal{L}$ is required at the central grid point $l=0$ :

$$
\begin{array}{r}
\mathfrak{l}_{0,0}(\alpha, \beta)=\frac{4}{N_{\theta}^{2} h_{r}^{4}} \sum_{p=0}^{N_{\theta}-1} \sum_{q=0}^{N_{\theta}-1}\left[\left(1-4 c_{p} c_{q}-4 s_{p} s_{q}\right) \alpha_{1, p} \beta_{1, q}\right. \\
\left.-2\left(\alpha_{1, p} \beta_{0,0}+\alpha_{0,0} \beta_{1, q}-\alpha_{0,0} \beta_{0,0}\right)\right]
\end{array}
$$

where $c_{l}=\cos \left(4 \pi l / N_{\theta}\right)$ and $s_{l}=\sin \left(4 \pi l / N_{\theta}\right)$.

The approximations $\delta_{\Delta}, \delta_{\Delta \Delta}$ and $\mathfrak{l}$ are second-order accurate in $r$ and $\theta$.

\section{B. An Implicit Scheme}

Given the above set of basic difference operators, a large number of schemes for the shell system (5) is possible. Here is a particularly simple family:

$$
\begin{aligned}
\left(1+g \kappa^{2} h_{t}^{2} \delta_{\Delta \Delta}\right) \delta_{t t} u & =-\kappa^{2}\left(\delta_{\Delta \Delta} u-\mathfrak{l}(\Phi, u)+q \delta_{\Delta} \Phi\right) \\
& -2 \sigma_{0} \delta_{t} \cdot u+2 \sigma_{1} \delta_{\Delta} \delta_{t} \cdot u+F \epsilon \\
\delta_{\Delta \Delta} \Phi= & -\mathfrak{l}(u, u)+q \delta_{\Delta} u
\end{aligned}
$$

This family is implicit, and depends on the free parameter $g$, which may be used in order to compensate for effects of numerical dispersion-see Section IV-A. The input function $F$ is now a time series, i.e., $F=F^{n}$, and $\epsilon=\epsilon_{l, m}$ is a specified grid function-see Section III-E for more on particular choices of these functions.

As written, the scheme above, when applied at grid points with $l=N_{r}$, or $l=N_{r}-1$ requires access to values at virtual grid points with $l=N_{r}+1$ and $l=N_{r}+2$, which are outside the domain of definition of the scheme; values at such points may be set through boundary conditions-see the next section. This scheme, as it is implicit, requires linear system solutions at each time step—see Section III-G.

\section{Numerical Boundary Conditions}

There are many choices of numerical edge conditions corresponding to (7); here is one choice

$$
\begin{array}{r}
\left(\delta_{\Delta}-(1-\nu) \delta_{r .}-(1-\nu) \delta_{\theta \theta}\right) u_{N_{r}, m}=0 \\
\left(\mu_{r-}\left(\left(\mu_{r+} r\right) \delta_{r+} \delta_{\Delta}\right)+(1-\nu)\left(\delta_{r .}-1\right) \delta_{\theta \theta}\right) u_{N_{r}, m}=0 .
\end{array}
$$

Such conditions allow the scheme (12) to be updated in terms of values over the interior of the unit circle.

Conditions corresponding to (8) for $\Phi$ are

$$
\Phi_{N_{r}, m}=0 \quad \delta_{r} . \Phi_{N_{r}, m}=0
$$

and thus values of the grid function $\Phi_{l, m}$ at $l=N_{r}$ need not be stored in implementation.

If the shell is assumed clamped near the center, over a circle of small radius, then simple boundary conditions corresponding to (9) are

$$
u_{0,0}=u_{1, m}=\Phi_{0,0}=\Phi_{1, m}=0
$$

and, as above, values of the grid functions at the center and first ring of grid points need not be stored in implementation.

\section{Numerical Stability Conditions}

Sufficient numerical stability conditions for scheme (12) are difficult to obtain. Necessary conditions, obtained by examining the behavior of the scheme under linear conditions, may be shown to be

$$
\begin{aligned}
& \frac{2 \kappa h_{t} \sqrt{1-4 g}}{h_{r}^{2} \sqrt{1-h_{t}^{2} \kappa^{2} q^{2} / 4}}\left(1+\frac{1}{h_{\theta}^{2}}\right) \leq 1 \quad \text { Free } \\
& \frac{2 \kappa h_{t} \sqrt{1-4 g}}{h_{r}^{2} \sqrt{1-h_{t}^{2} \kappa^{2} q^{2} / 4}}\left(1+\frac{1}{4 h_{\theta}^{2}}\right) \leq 1 \quad \text { Clamped }
\end{aligned}
$$

when $g<1 / 4$, which is the case of most interest with regard to the maximization of output bandwidth-see Section IV-A. Either of the relations above implies, for a given choice of the time step $h_{t}$, a range of allowable values for the grid spacings $h_{r}$ and $h_{\theta}$. In practice, it is easiest to work with the quantities $h_{r}$ and the radial grid aspect ratio $\xi=h_{\theta} / h_{r}$. For a given choice of $\xi$, the above relations may be written explicitly as

$$
h_{r} \geq h_{r, \min }\left(\kappa, q, g, h_{t}, \xi\right)
$$

which is a Courant-Friedrichs-Lewy type lower bound [25] on the grid spacing. It is always a good idea to choose $h_{r}$ as close to this bound as possible [16], though $h_{r}$ must normally be chosen slightly away from this bound due to grid truncation effects (i.e., $h_{r}=1 / N_{r}$, for some integer $N_{r}$ ). Once $h_{r}$ has been chosen, the number of grid points $N_{\theta}$ in the angular direction may be chosen as $N_{\theta}=$ floor $\left(2 \pi / \xi h_{r}\right)$. A good range of choices of the aspect ratio $\xi$ is between 2 and 5 .

Necessary conditions such as the above may be shown to be sufficient in the fully nonlinear case, at least in the case of plates, when a slightly different form of the discrete operator $\mathfrak{l}$ is used-see [26]. The same is presumably true in the present case, though an altered form of $\mathfrak{l}$ complicates implementation 

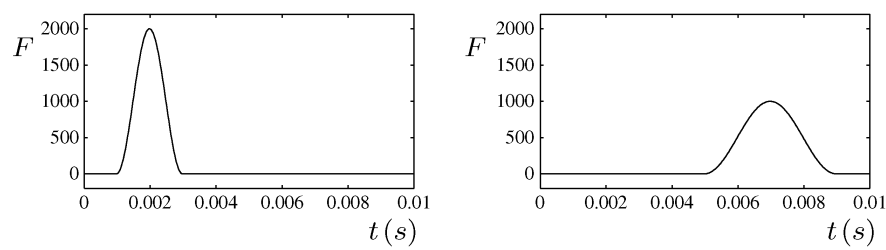

Fig. 3. Excitation functions $F$, of the form given in (16). (a) With parameters $F_{\max }=2000, t_{\mathrm{exc}}=2 \mathrm{~ms}$, and $t_{0}=0.001 \mathrm{~s}$, and (b) with parameters $F_{\text {max }}=1000, t_{\text {exc }}=4 \mathrm{~ms}$, and $t_{0}=0.005 \mathrm{~s}$.

somewhat. For this reason, it is perhaps best to stick with the simple scheme as given here. As a rule of thumb, numerical instability begins to manifest itself when vibration amplitudes approach the limit of validity of this particular shell model (i.e., when the vibration amplitudes, in dimensional form, are several times the shell thickness).

\section{E. Excitation Functions}

Excitation of percussion instruments is generally carried out through a striking mechanism (such as a mallet). In a complete physical model, the mallet should be modelled separately, and its interaction with the shell system will give rise to the term involving $F$ in (5). Given, though, that interaction times are extremely short (on the order of between 1 and $20 \mathrm{~ms}$ ), a useful synthesis shortcut is to simply specify $F$ as a given function; here is one parameterized set:

$$
F(t)= \begin{cases}\frac{F_{\max }}{2}\left(1-\cos \left(\frac{2 \pi\left(t-t_{0}\right)}{t_{\mathrm{exc}}}\right)\right), & t_{0} \leq t \leq t_{0}+t_{\mathrm{exc}} \\ 0, & \text { otherwise. }\end{cases}
$$

Here, $F_{\max }$ is the maximum of the excitation, which is of duration $t_{\mathrm{exc}}$, and occurs at time $t=t_{0}$. See Fig. 3. In general, when related to a function $F(t)$ which results from coupling to a mallet model, $F_{\max }$ increases, and $t_{\text {exc }}$ decreases as strike strength increases.

For the spatial distribution, given the grid is usually quite coarse, it is probably sufficient to make use of an impulse-like grid function of the form

$$
\epsilon_{l, m}=\frac{1}{l h_{r}^{2} h_{\theta}} \delta_{l_{0}, m_{0}}
$$

where $\delta_{l_{0}, m_{0}}$ is a Kronecker delta function, selecting the location $l=l_{0}, m=m_{0}$, corresponding to a spatial location $r=l h_{r}, \theta=m h_{\theta}$. It is not difficult to extend this definition to a distribution of finite width-see [16].

\section{F. Output}

From the point of view of musical acoustics, the radiation characteristics of a percussion instrument depend on geometry in a complex manner [4]. The rigorous generation of output, from a time domain scheme such as (12), would require a sum over paths from all points on the surface of the resonator to the listening point, perhaps incorporating effects of the enclosure (i.e., the room) itself. For synthesis, however, once the effects of radiation losses have been incorporated into the model, it is

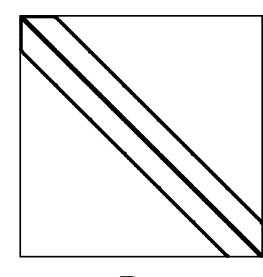

$\mathbf{D}_{\Delta}$

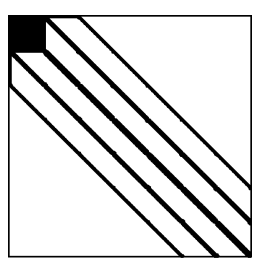

$\mathbf{D}_{\Delta \Delta}$
Fig. 4. Sparsity patterns for the matrix representations of the discrete Laplacian $\mathbf{D}_{\Delta}$ and bi-Laplacian operator $\mathbf{D}_{\Delta \Delta}$, under a free center condition.

much simpler to simply read output velocity from a single point on the surface of the resonator, or, in the present context, from a value computed on the grid at a given location, i.e.,

$$
v_{\mathrm{out}}=\delta_{t-} u_{l_{\mathrm{out}}, m_{\mathrm{out}}}^{n}
$$

where $l_{\text {out }}$ and $m_{\text {out }}$ are grid indices, and where $\delta_{t-}$ is a simple backward difference. Though there are subtle spectral effects associated with the choice of output location, generally, the choice of an output location near the shell edge leads to an emphasis on high frequencies. For synthesis purposes, it is probably simplest to adjust this, if desired, using rudimentary filtering applied at a supplementary step to the output signal $v_{\text {out }}$.

One of the interesting features of time-domain methods is that multiple outputs may be generated essentially at no extra cost, as the entire state is directly observable at each time step (this is not true, e.g., of modal methods, which require a separate expansion at each output location). As such, as a crude means of spatializing sound output, one could draw separate audio channels from distinct locations, which could even be moving, similarly to the case of scanned synthesis [27], and thus requiring some form of interpolation. For more on this, see [16].

\section{G. Implementation Details}

In implementation, it is useful to rewrite an implicit scheme such as (12) in a vector-matrix update form. A given grid function $f_{l, m}^{n}$ may be rewritten as a vector $\mathbf{f}^{n}$, by stacking consecutive concentric rings, as

$$
\mathbf{f}^{n}=\left[f_{0,0}^{n}, f_{1,0}^{n}, \ldots, f_{1, N_{\theta}-1}^{n}, \ldots, f_{N_{r}, 0}^{n} \ldots, f_{N_{r}, N_{\theta}-1}^{n}\right]^{T} .
$$

(If the center point is clamped, then the center value of the grid function and those at the first ring may be neglected.) The operators $\delta_{\Delta}$ and $\delta_{\Delta \Delta}$ are linear, and thus may be written, in terms of their operation on grid functions, as matrix multiplications $\mathbf{D}_{\Delta}$ and $\mathbf{D}_{\Delta \Delta}$, once the boundary conditions described in Section III-C have been incorporated-note that the exact forms and sizes of these matrices will depend on the type of boundary and center condition. Such forms are particularly sparse-see Fig. 4, showing sparsity patterns for these matrices under free center and boundary conditions.

Scheme (12) then takes the form

$$
\begin{aligned}
\mathbf{A} \mathbf{u}^{n+1}= & \mathbf{B u}^{n}+\mathbf{C} \mathbf{u}^{n-1}+\kappa^{2} h_{t}^{2} \mathfrak{l}\left(\boldsymbol{\Phi}^{n}, \mathbf{u}^{n}\right) \\
& -\kappa^{2} q h_{t}^{2} \mathbf{D}_{\Delta} \Phi^{n}+h_{t}^{2} F^{n} \boldsymbol{\epsilon} \\
\mathbf{D}_{\Delta \Delta} \boldsymbol{\Phi}^{n}= & -\mathfrak{l}\left(\mathbf{u}^{n}, \mathbf{u}^{n}\right)+q \mathbf{D}_{\Delta} \mathbf{u}^{n}
\end{aligned}
$$


where

$$
\begin{aligned}
& \mathbf{A}=\left(1+\sigma_{0} h_{t}\right) \mathbf{I}+g \kappa^{2} h_{t}^{2} \mathbf{D}_{\Delta \Delta}-\sigma_{1} h_{t} \mathbf{D}_{\Delta} \\
& \mathbf{B}=2 \mathbf{I}+\kappa^{2} h_{t}^{2}(2 g-1) \mathbf{D}_{\Delta \Delta} \\
& \mathbf{C}=-\left(1-\sigma_{0} h_{t}\right) \mathbf{I}-g \kappa^{2} h_{t}^{2} \mathbf{D}_{\Delta \Delta}-\sigma_{1} h_{t} \mathbf{D}_{\Delta}
\end{aligned}
$$

and where $\mathbf{I}$ indicates an identity matrix of the appropriate size. Note that the instances of $\mathbf{D}_{\Delta}$ and $\mathbf{D}_{\Delta \Delta}$ above are not all identical, as boundary conditions are distinct depending on whether the operator is applied to $\mathbf{u}$ or $\boldsymbol{\Phi} . \boldsymbol{\epsilon}$ is the excitation distribution $\epsilon_{l, m}$ rewritten similarly in vector form.

Given that values of the grid function $\mathbf{u}^{n}$ have been computed through time step $n$, then two linear system solutions are required in order to perform an update: first, (18b) may be solved for $\boldsymbol{\Phi}^{n}$, after an evaluation of the nonlinear term $\mathfrak{l}\left(\mathbf{u}^{n}, \mathbf{u}^{n}\right)$. Given $\boldsymbol{\Phi}^{n}$, the nonlinear term in (18a) may be evaluated, and a linear system solved in order to yield $\mathbf{u}^{n+1}$, after which the cycle is repeated.

Computation time is determined by the shell parameters $\kappa$ and $q$, the sample rate, and the scheme free parameter $g$. Given that the grid spacings are bounded by a stability condition such as (15), which should be satisfied as close to equality as possible, it is then possible to estimate the total number of grid points $\mathcal{N} \approx N_{\theta} N_{r}$ as

$$
\mathcal{N} \approx \pi \sqrt{\frac{2}{\kappa h_{t}}}\left(\frac{1-h_{t}^{2} \kappa^{2} q^{2}}{1-4 g}\right)^{1 / 4}
$$

Thus, the grid size increases with the square root of the sample rate, is decreased as curvature increases, and increases as the parameter $g$ approaches 1/4 (optimal values of $g$, with respect to numerical dispersion are close to 1/4). Typical values of $\mathcal{N}$, for shell-like systems in musical acoustics, and at a typical audio sample rate such as $44.1 \mathrm{kHz}$, lie in the range between $\mathcal{N}=100$ and $\mathcal{N}=500$, and are thus not extremely large.

The main computational costs, then, in an implementation of scheme (12) can be seen to be, from the update form (18), linear system solutions involving the matrices $\mathbf{A}$ and $\mathbf{D}_{\Delta \Delta}$, which are of size $\mathcal{N} \times \mathcal{N}$. It is important to note that because the various difference operators are extremely sparse in matrix form (see Fig. 4), with $O(\mathcal{N})$ nonzero entries, such solutions may be performed in $O(\mathcal{N})$ operations (using, e.g., an iterative method). Various specialized linear system solution techniques may be used - for block-banded matrices, the Thomas algorithm [28] is often employed. Given that the matrices also exhibit a good deal of structure, then methods based on block Toeplitz inversion, perhaps employing the fast Fourier transform (FFT) are also a possibility. Notice that even though this is a nonlinear system, only linear system solutions involving constant matrices (i.e., $\mathbf{D}_{\Delta \Delta}$ and $\mathbf{A}$ ) are required; thus if one were to use standard iterative methods [25], there is the possibility of performing preconditioning and factorization operations before run-time.

\section{Simulation Results}

The results of using scheme (12) are as expected (see Fig. 5), showing snapshots of the time evolution of the displacement of shell when subject to a localized initial distribution. In audio

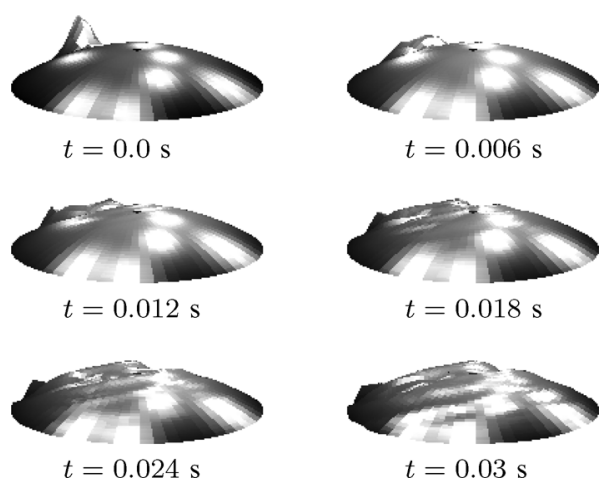

Fig. 5. Snapshots of the time evolution of the state of a spherical shell, according to scheme (12), at times as indicated. The shell has parameters $\kappa=1$ and $q=60$, is under a center clamped boundary condition, and is initialized with a localized displacement. The sample rate is $40 \mathrm{kHz}$.

TABLE I

Modal Frequencies, in Hz, For a Circular Plate, With $\kappa=20$ AND $\nu=0.3$, UNDER FREE EdGE AND CENTER CONDITIONS. EXACT VALUES, VALUES COMPUTED BY SCHEME (12), RUNNING AT $44.1 \mathrm{kHz}$, With $g=0$, AND VAlues COMPUTED With $g=0.2498$ ARE GiVEn, With

ZERO-FREQUENCY (RIGID BODY) AND DEGENERATE MODES IGNORED

\begin{tabular}{|c||c|c|c|}
\hline Mode \# & Exact Freq. $(\mathrm{Hz})$ & $\mathrm{g}=0$ & $\mathrm{~g}=0.2498$ \\
\hline 1 & 17.04 & 16.32 & 16.93 \\
2 & 28.66 & 26.72 & 28.39 \\
3 & 39.57 & 37.07 & 39.17 \\
4 & 65.03 & 59.53 & 64.37 \\
5 & 69.48 & 63.54 & 68.47 \\
\hline
\end{tabular}

applications, however, most interesting features are best exhibited using spectral plots and spectrograms. In the following section, all spectra are obtained through the DFT of the output signal $v_{\text {out }}$, written as $V(f)$, and shown as magnitude plots in $\mathrm{dB}$. Spectrograms are obtained by using the short-time Fourier transform, using a window size of 1024 points, and an overlap of 128 points, and using Hanning windows. They are also plotted in $\mathrm{dB}$.

\section{A. Numerical Dispersion, Bandwidth, and Tuning}

Schemes in radial coordinates are naturally much more dispersive than schemes in Cartesian coordinates, due to variations in the spacings between points on the grid-there are two sideeffects of numerical dispersion which are of great importance in sound synthesis applications: modal detuning, and bandwidth limitation. The free scheme parameter $g$ allows a convenient means of reducing both such effects. (It is important to note that regardless of the value of $g$, scheme (12) is consistent with the underlying shell system - so in the limit of a high sample rate, and when the scheme is stable, computed values will tend towards those of the model system, for any $g$.)

Consider first a comparison between computed modal frequencies for scheme (12), with $g=0$, and for a nonzero value of $g$, as shown in Table I, for a flat circular plate with $\kappa=20$. For $g=0$, deviations on the order of approximately a semitone are apparent beyond the fifth partial. For $g=0.2498$, the 

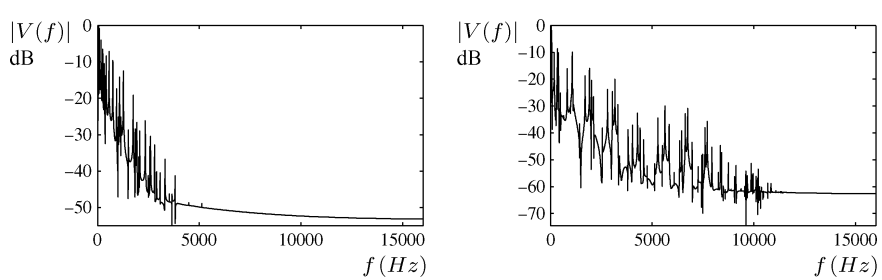

Fig. 6. Output spectra $|V(f)|$, in $\mathrm{dB}$, for scheme (12), for a shell with $\kappa=100$, $q=5$, and under free center conditions, under low-amplitude vibration (i.e., linear) conditions. At left, the tuning parameter $g=0$, and at right, $g=0.249$. The sample rate is $32 \mathrm{kHz}$. Note the significantly wider bandwidth in the case of the parameterized scheme, where $g$ has been adjusted to maximize bandwidth, and minimize numerical dispersion.

computed frequencies suffer much less of a degradation in accuracy. In general, such detuning will decrease as the sample rate is raised. As mentioned previously, the best results are obtained when a stability condition such as (15) are satisfied as close to equality as possible.

Related to this detuning is the much more severe problem of global reduction in bandwidth for a poorly designed scheme, as shown in the case of the scheme with $g=0$, in Fig. 6(a)-this is a common occurrence in schemes defined over nonuniform grids (such as in the present case of radial coordinates) [16]. As such, the results are not satisfactory, perceptually, and furthermore, in the nonlinear case, effects which rely on large audio bandwidth, such as crashes, will be strongly curtailed. The benefits of parameterized schemes, in mainstream applications, are often viewed in terms of computational efficiency - a larger time step may be chosen for a given grid spacing. In audio applications, however, the time step is fixed, and the benefit is rather in terms of greater allowed bandwidth (and much reduced numerical dispersion as discussed above), as shown in Fig. 6(b).

In general, in scheme (12), a good choice of the tuning parameter $g$ is at a value slightly less than $1 / 4$, or at the edge of the region for which the scheme is conditionally stable in the linear case. See, e.g., [12]. As is always the case in time domain methods for time-dependent problems, the greatest output bandwidth is obtained when the grid spacings are chosen to satisfy a CFL-type stability condition (in this case, (15)) as close to equality as possible. On the other hand, for $g$ near $1 / 4$, the optimal grid spacings $h_{r}$ and $h_{\theta}$, from the stability conditions (15), are smaller, and there is thus an increase in computational cost. This increase in cost may be interpreted in terms of the number of degrees of freedom required to adequately represent the solution to (5) at a given sample rate, which is too small when an explicit scheme is employed [16].

\section{B. Shell Curvature and Mode Locations}

Remaining with the assumption of linear behavior, it is useful to examine the effect of curvature on the resulting output spectrum. The modal frequencies depend strongly on this curvature-in general, the frequencies of asymmetric modes without nodal circles vary only slightly with curvature, whereas the frequencies of all other modes exhibit a sharp increase [18] (see Fig. 7), which illustrates this shifting with increased values of
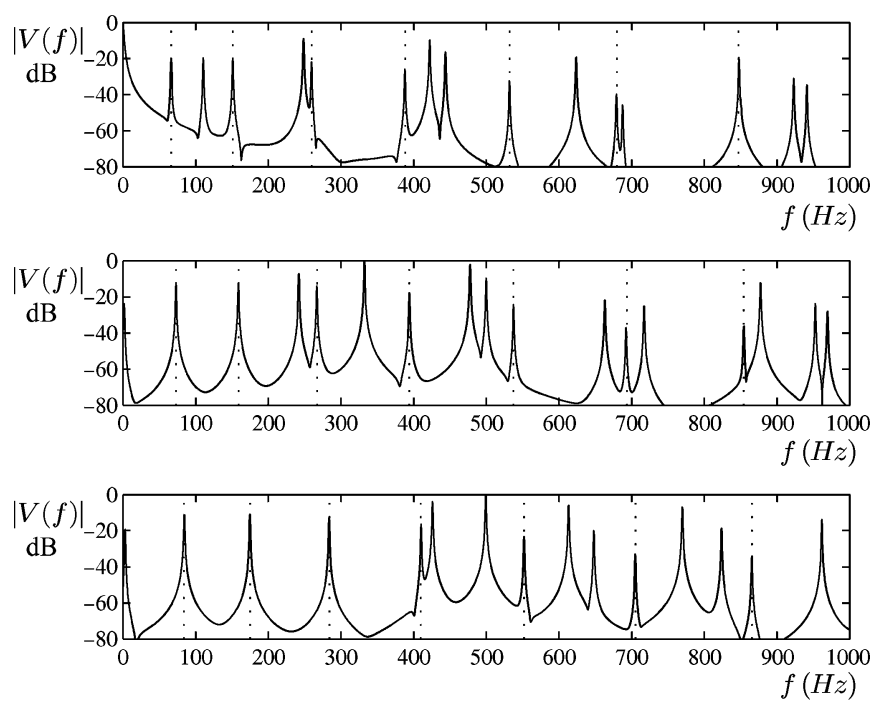

Fig. 7. Output magnitude spectra, $|V(f)|$, in $\mathrm{dB}$, for the shell model (5) under free center conditions, using scheme (12), running at $44.1 \mathrm{kHz}$, for $\kappa=80$, and for increasing values of the curvature parameter $q=0$ (top), $q=20$ (middle), and $q=40$ (bottom). Modal frequencies corresponding to asymmetric modes without nodal circles are indicated by dotted lines. Such modes are little affected by a change in curvature, unlike the remaining modes, which experience a strong upward shift in frequency with increased curvature.

the curvature parameter $q$, for a fixed value of $\kappa$, the stiffness parameter. Perceptually, increased curvature leads to an increased density of modes in the mid range of the audio spectrum, and a resulting dissonance, characteristic of cymbal-like sounds.

\section{Pitch Glides}

Perhaps the simplest example of nonlinear behavior in the shell model (5) is the pitch glide, a common feature across many percussive instruments. The modeling of pitch glides, for synthesis, has been approached in the case of strings and membranes through a variety of methods [11], [29], [30]. Usually the pitch glide is downward, from the moment of impact excitation, reflecting stiffening behavior of the medium at higher vibration amplitudes (though upward pitch glides are also possible [4]). See Fig. 8.

Clearly, loss plays a much greater role, perceptually, in the nonlinear case-not only is the global decay profile determined by the loss parameters, but the precise trajectory of the glide itself. Pitch glides are most easily heard when the parameter $\kappa$ is relatively high (i.e., for high-pitched structures). For lower values of $\kappa$, the pitch glide effect is subsumed by a dominant crash effect—see the next section.

\section{Crashes}

Crashes are one of the primary results of the use of a nonlinear model, and the effects on synthetic output are dramatic-there is a slow build-up of high-frequency energy in the period following a strike (often over several hundred milliseconds, or longer), and the individual harmonics undergo bifurcations, leading to a noise-like sound output. See Fig. 9, which illustrates this effect, for excitations of increasing intensity. Beyond the dependence of the output on the strength of 

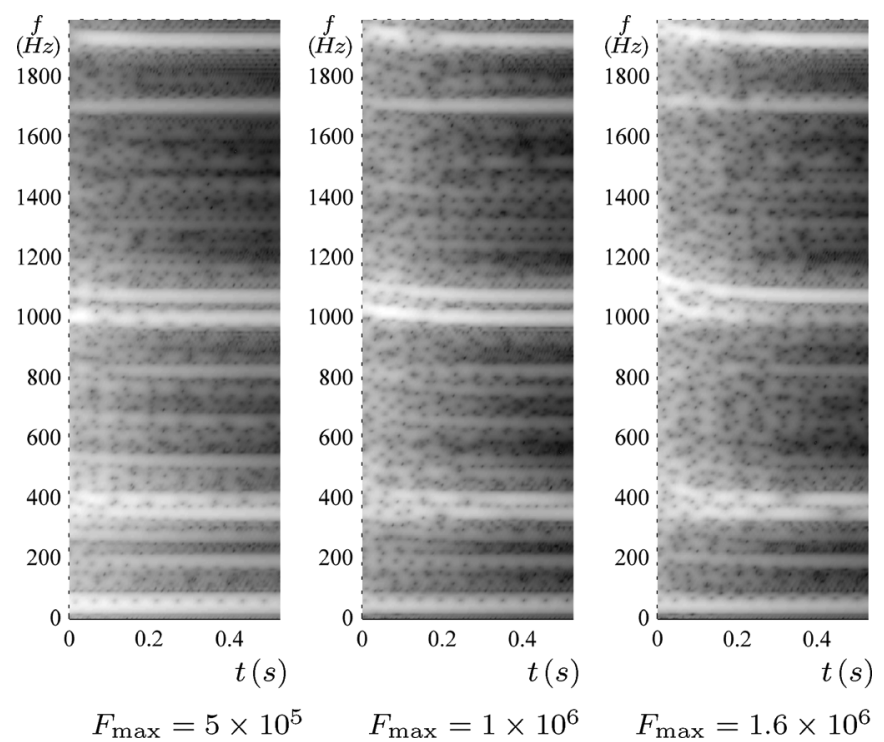

Fig. 8. Spectrograms of sound output from scheme (12), running at $32 \mathrm{kHz}$, illustrating pitch glide phenomena. Here, the shell has $\kappa=100, q=5$, and loss parameters $\sigma_{0}=4.58$ and $\sigma_{1}=0.004$, corresponding roughly to a $60-\mathrm{dB}$ decay time of $3 \mathrm{~s}$, and a clamped center condition. The excitation, of the form (16), is applied at time $t_{0}=0$, is of duration $t_{\mathrm{exc}}=2 \mathrm{~ms}$, and is of strength $F_{\max }$ as given above. Generally, the pitch glide effect becomes greater under stronger excitations.
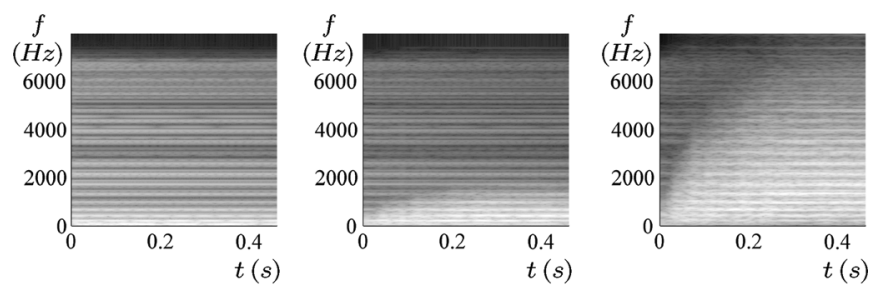

$F_{\max }=3 \times 10^{4}$

$$
F_{\max }=1 \times 10^{5}
$$

$F_{\text {max }}=3 \times 10^{5}$

Fig. 9. Spectrograms of sound output from scheme (12), running at $32 \mathrm{kHz}$, for a shell with $\kappa=20$ and $q=5$, under a clamped center condition. An excitation function of the form given in (16) is applied at time $t_{0}=0$, and over a duration of $t_{\mathrm{exc}}=16 \mathrm{~ms}$, at a location at $r=0.8$. The strength $F_{\max }$ of the excitation is as indicated above. In the final plot, the gradual increase in high frequency energy over $200-300 \mathrm{~ms}$ is easily visible.

the exitation, there is an additional complex dependence on the location of the excitation, as well as on the duration.

Because of the spontaneous generation of high-frequency energy, the model of frequency-dependent loss included in (5) again plays an important role in the nonlinear case. The cascade of high-frequency energy is increasingly attenuated at high frequencies (see the expression for the frequency-dependent decay time $T_{60}(f)$ given in (6)), having a great impact on the resulting amplitude envelope-see Fig. 10. A good model of frequency-dependent loss is also extremely important for numerical reasons-if it is not present, aliasing phenomena can be observed as energy approaches the Nyquist frequency.

\section{E. Gestures}

As an example of a crude musical use of this algorithm, consider a striking gesture, consisting of a series of equally-spaced impulses, corresponding to a percussive "roll," as illustrated at top in Fig. 11. The application of such an excitation to a
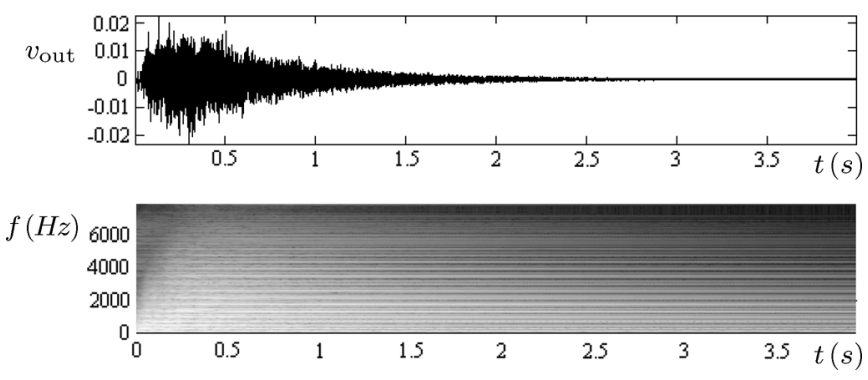

Fig. 10. Top, variation in the time envelope of sound output $v_{\text {out }}$ from (12), and its spectrogram, under the conditions as given in the third panel of Fig. 9 above. The loss parameters are $\sigma_{0}=1.34$ and $\sigma_{1}=0.0012$.
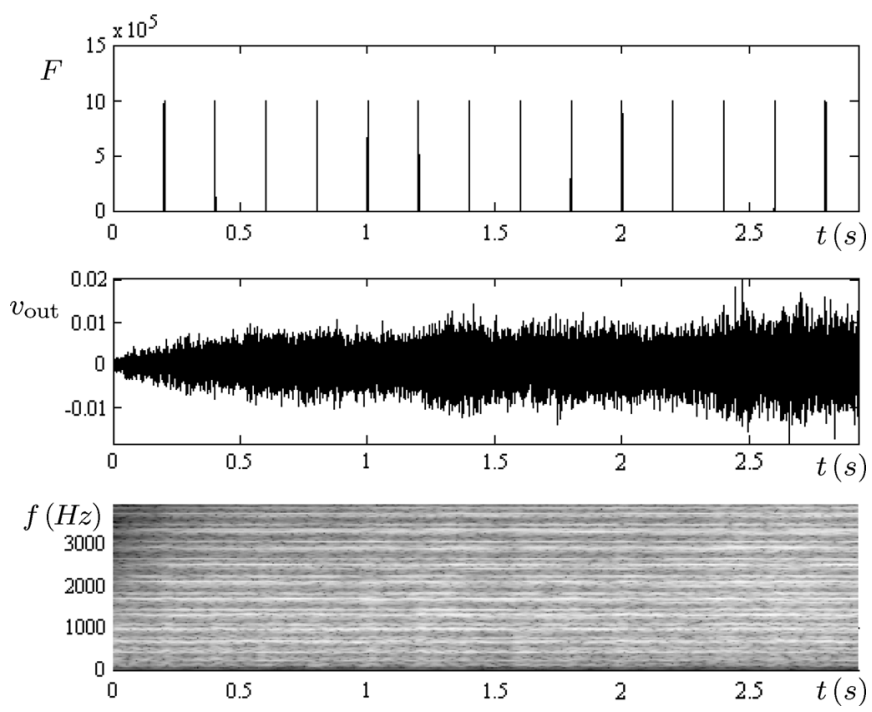

Fig. 11. Top: a simple excitation gesture, made up of individual pulses of the form given in (16), with $F_{\max }=10^{6}$, and $t_{\mathrm{exc}}=3 \mathrm{~ms}$, spaced apart by 0.2 s. Middle: sound output $v_{\text {out }}$ from scheme (12), running at $32 \mathrm{kHz}$, for a shell with $\kappa=20$, and $q=30$, and under center-clamped conditions, under the application of the excitation at top, and bottom, its spectrogram. The general effect is of a pitched sound, with an underlying noise component.

linear model of a shell would lead, by superposition, to a repetitive pattern; when applied to a nonlinear model, however, extremely irregular variations in the output envelope result, and there is a gradual increase in the noise component, as illustrated in the middle and bottom panels of Fig. 11. This sound example, among others, is available at the Web location noted at the end of the Introduction.

\section{CONCLUSION}

The scheme (12), characterized by very few parameters, and subjected to very simple excitations, is capable of generating a rich variety of sounds very much characteristic of percussion instruments; the key to this interesting behavior is the strong nonlinearity present in the model. Time-domain methods such as finite-difference schemes are an excellent means of capturing this behavior, which has not been attempted to date using other synthesis techniques. Computational cost is high, but not extreme-all of the examples presented in this article were generated in Matlab, using standard laptops and workstations, with computation times on the order of between $1 \mathrm{~s}$ and $160 \mathrm{~s}$ per second of audio output, depending on the choice of model 
parameters and the audio sample rate. Computation times would be obviously much faster in a $\mathrm{C}$ implementation. Though some work at the level of algorithm design is necessary in order to obtain an algorithm which produces acceptable audio results (namely in order to reduce effects of numerical dispersion, which are particularly problematic for schemes defined in radial geometries), the runtime loop consists of a pair of linear system solutions, and matrix multiplications.

The model presented here is very basic - there are many opportunities for improvement. The model of the supporting system is very crude; a clamped center condition is a first approximation, but only that-a pivoting condition could also be desirable under some conditions, in which case effects of gravity will intervene, leading to a slow pendulum-like motion of a struck shell. In the case of cymbals, it may be necessary to model interaction between the shell and a support structure of finite dimensions (such as a ring-shaped damper), perhaps allowing for distributed collisions. In the case of gongs, though there is not a central support, the structure as a whole is necessarily suspended by ropes or wires, and thus pointwise tension is applied at the boundary, and the effects of gravity will again become evident. Though structures used as percussion instruments are often lightly curved and of relatively uniform thickness, most real instruments (such as gongs with a raised central dome, or cymbals of thickness which tapers towards an edge) do exhibit some such variation, in which case a more general model of shell vibration may be necessary [17]. Finally, the model of loss as presented here is qualitatively correct (as loss effects generally become stronger at high frequencies), and leads to as relatively simple implementation, but more refined modeling, perhaps along the lines of work carried out in the case of plates [22], may be desirable. Other extensions might involve the coupling to a mallet model [31], which is relatively straightforward, and perhaps the addition of other connected elements such as, in the case of cymbals, sizzles, which are rattling elements affixed to the shell near its rim.

\section{REFERENCES}

[1] J. O. Smith, III, Physical Audio Signal Processing Stanford, CA, 2004 [Online]. Available: http://ccrma.stanford.edu/ jos/pasp04/, Draft version

[2] J.-M. Adrien, "The missing link: Modal synthesis," in Representations of Musical Signals, G. DePoli, A. Picialli, and C. Roads, Eds. Cambridge, MA: MIT Press, 1991, pp. 269-297.

[3] A. Chaigne and J. Kergomard, Acoustique des Instruments de Musique. Paris, France: Belin, 2008.

[4] N. Fletcher and T. Rossing, The Physics of Musical Instruments. New York: Springer-Verlag, 1991.

[5] J. Laird, "The physical modeling of drums using digital waveguides," Ph.D. dissertation, University of York, , 2001.

[6] S. van Duyne, "Digital Filter Applications to Modeling Wave Propagation in Springs, Strings, Membranes and Acoustical Space," Ph.D. dissertation, Music Dept., Stanford Univ., , 2007.

[7] S. van Duyne and J. O. Smith, III, "Physical modelling with the 2D digital waveguide mesh," in Proc. Int. Comput. Music Conf., Tokyo, Japan, Sep. 1993, pp. 40-47.

[8] F. Fontana and D. Rocchesso, "Physical modelling of membranes for percussion instruments," Acta Acustica United With Acustica, vol. 84, no. 3, pp. 529-542, 1998.
[9] S. van Duyne and J. O. Smith, III, "A simplified approach to modelling dispersion caused by stiffness in strings and plates," in Proc. Int. Comput. Music Conf., Århus, Denmark, Sep. 1994, pp. 407-410.

[10] L. Trautmann and R. Rabenstein, Digital Sound Synthesis by Physical Modeling Using the Functional Transformation Method. New York: Kluwer/Plenum, 2003.

[11] S. Petrausch and R. Rabenstein, "Tension modulated nonlinear 2D models for digital sound synthesis with the functional transformation method," in Proc. EUSIPCO'05, 13th Eur. Signal Process. Conf., Antalya, Turkey, Sep. 2005.

[12] K. Arcas, "Simulation numérique d'un réverbérateur à plaque," $\mathrm{Ph} . \mathrm{D}$. dissertation, Ecole Nationale Supérieure de Techniques Avancées, Palaiseau, France, 2008.

[13] N. Giordano and M. Jiang, "Physical modeling of the piano," EURASIP J. Appl. Signal Process., vol. 2004, no. 1, pp. 926-933, 2004.

[14] S. Bilbao, K. Arcas, and A. Chaigne, "A physical model of plate reverberation," in Proc. IEEE Int. Conf. Acoust., Speech, Signal Process., Toulouse, France, 2006, vol. 5, pp. 165-168.

[15] S. Bilbao, "Sound synthesis for nonlinear plates," in Proc. 11th Int. Digital Audio Effects Conf., Madrid, Spain, Sep. 2005, pp. 243-248.

[16] S. Bilbao, Numerical Sound Synthesis. Chichester, U.K.: Wiley, 2009, In Press.

[17] A. Leissa, Vibration of Shells. Melville, NY: Acoustical Society of America, 1993.

[18] O. Thomas, C. Touzé, and A. Chaigne, "Non-linear vibrations of freeedge thin spherical shells: Modal interaction rules and 1:1:2 internal resonance," Int. J. Solids Structures, vol. 42, pp. 3339-3373, 2005.

[19] R. Szilard, Theory and Analysis of Plates. Englewood Cliffs, NJ: Prentice-Hall, 1974.

[20] A. Nayfeh and D. Mook, Nonlinear Oscillations. New York: Wiley, 1979.

[21] K. Graff, Wave Motion in Elastic Solids. New York: Dover, 1975.

[22] S. Schedin, C. Lambourg, and A. Chaigne, "Transient sound fields from impacted plates: Comparison between numerical simulations and experiments," J. Sound Vibr., vol. 221, no. 32, pp. 471-490, 1999.

[23] C. Touzé, O. Thomas, and A. Chaigne, "Asymmetric nonlinear forced vibrations of free-edge circular plates. Part I. Theory," J. Sound Vibr., vol. 258, no. 4, pp. 649-676, 2002.

[24] G. Evans, J. Blackledge, and P. Yardley, Numerical Methods for Partial Differential Equations. London, U.K.: Springer, 1999.

[25] J. Strikwerda, Finite Difference Schemes and Partial Differential Equations. Pacific Grove, CA: Wadsworth and Brooks/Cole , 1989.

[26] S. Bilbao, "A family of conservative finite difference schemes for the dynamical von Karman plate equations," Numerical Methods for Partial Differential Equations, vol. 24, no. 1, pp. 193-216, 2008.

[27] W. Verplank, M. Mathews, and R. Shaw, "Scanned synthesis," in Proc. Int. Comput. Music Conf., Berlin, Germany, Aug. 2000, pp. 368-371.

[28] L. H. Thomas, Watson Scientific Comput. Lab., Columbia Univ., "Elliptic problems in linear difference equations over a network," New York, 1949, Tech. Rep..

[29] S. Bilbao, "Energy-conserving finite difference schemes for tensionmodulated strings," in Proc. IEEE Int. Conf. Acoust., Speech, Signal Process., Montreal, QC, Canada, May 2004, vol. 4, pp. 285-288.

[30] V. Välimäki, T. Tolonen, and M. Karjalainen, "Plucked-string synthesis algorithms with tension modulation nonlinearity," in Proc. IEEE Int. Conf. Acoust., Speech, Signal Process., Phoenix, AZ, Mar. 1999, vol. 2, pp. 977-980.

[31] L. Rhaouti, A. Chaigne, and P. Joly, "Time-domain modeling and numerical simulation of a kettledrum," J. Acoust. Soc. Amer., vol. 105, no. 6, pp. 3545-3562, 1999.

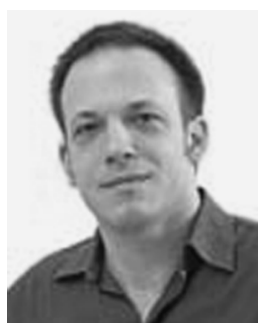

Stefan Bilbao received the B.A. degree in physics from Harvard University, Cambridge, MA, in 1992 and the M.Sc. and Ph.D. degrees in electrical engineering from Stanford University, Stanford, CA, in 1996 and 2001, respectively.

$\mathrm{He}$ is currently a Senior Lecturer in the Acoustics and Fluid Dynamics Group/Music subject area, University of Edinburgh, Edinburgh, U.K., and was previously a Lecturer at the Sonic Arts Research Centre, Queen's University Belfast, and a Research Associate at the Stanford Space Telecommunications and Radioscience Laboratories. 